\title{
Bi-orthogonal Wavelet based Adaptive Image Watermarking using Human Visual System and Fuzzy Inference System
}

\author{
Vaishali S. Jabade \\ Assistant Professor \\ Vishwakarma Institute of Technology, Pune, India
}

\author{
Sachin R. Gengaje, Ph.D. \\ Professor \\ Walchand Institute of Technology, Solapur, India
}

\begin{abstract}
Digital age today has shown increasing reliance on digital media and rapid distribution of digital contents. The issues of piracy and copyright are of immense concern in this exponentially increasing digital revolution. In this paper, authors propose use of bi-orthogonal wavelet transform as an effective tool to address copyright infringement issues. A synergistic combination of bi-orthogonal wavelet transform, human visual system characteristics and fuzzy inference system is arrived at to develop a novel adaptive image watermarking scheme. Logo watermark is used to prove ownership of digital property. A hierarchical data structure is generated using bi-orthogonal wavelet decomposition to represent an image. The reconstruction of image is achieved by computing inverse wavelet transform of modified wavelet coefficients. The unique feature of the proposed scheme is that fuzzy inference system is used to effectively establish relation between different HVS characteristics and adaptively adjusting embedding strength of watermark.
\end{abstract}

\section{General Terms}

Image Watermarking

\section{Keywords}

Bi-orthogonal Wavelet Transform, Human Visual System (HVS), Fuzzy Inference System (FIS)

\section{INTRODUCTION}

Internet facilitates publication, use and transfer of diversified digital information. The digital information includes images, text, music, pictures, video etc. To protect the interest of the creator, it is imperative to develop protection mechanism to avoid illegitimate copy, modification, tempering or forging of digital creations. In this digitized world, it is a challenge to protect ownership of digital data. In this context, copyright protection of digital images has received widespread attention in recent years [1].

Digital watermarking is an effective tool for protecting multimedia data from such illegitimate copying. The process of image watermarking comprises aspect of altering the pixel values of an image in a manner so that there is only a slight difference between original image and watermarked image [2]. This difference may be perceptually visible or invisible. Such a change may be detected by machines. The watermarks or copyright protection are selected in the form of date, serial number, trademark, logos or any such copyright information relating to the respective host image that is to be protected. One can extract this information as watermark in order to verify originality and establish ownership of digital content [3]. Most of the conventional watermarking techniques reported in literature embed pseudo-random sequence as watermark. This technique suffers from limitation that in spite of extraction of these watermarks it is very difficult to distinctively identify it and prove ownership of creator [4].

It is necessary to develop an easily readable and identifiable watermark to be embedded in the host images. The novel method discussed in this paper enables image owner or creator to adaptively adjust strength of watermark through fuzzy inference system, so that robustness of watermark can be enhanced [5-9].

\section{IMAGE WATERMARKING REQUIREMENTS}

Digital image watermarking attributes differ depending on its application. Following attributes characterize image watermarking for diversified applications [10-14].

\subsection{Imperceptibility}

This attribute represents transparency of watermark such that it is perceptually visible or invisible. It is important that modification of host image is inconspicuous after watermarks are embedded.

\subsection{Robustness}

The watermark needs to be robust enough against various intentional or non-intentional attacks on the host image Watermark in image must be resistant to signal processing attacks and geometric attacks. Signal processing attacks include filtering, JPEG compression, addition of salt and pepper noise, Gaussian noise etc. Geometric attacks include rotation, scaling, cropping etc. Robust watermarks should sustain most of these attacks and are useful for copyright applications.

\subsection{Blindness}

Blindness refers to the ability to recover copyright information with or without the use of host image. The copyright protection scheme can be classified as blind (oblivious) or non-blind (non-oblivious). In blind or private scheme, original host image is not required to extract watermark while in non-blind or public scheme requires host image for copyright information extraction.

\subsection{Un-ambiguity}

It refers to clear verification of extracted watermark. The extracted logo must be clear enough so that it can indicate ownership of the host image without any ambiguity.

\section{PROPOSED SCHEME}

The proposed approach is based on aspects of bi-orthogonal wavelet transform, HVS and FIS. 


\subsection{Bi-orthogonal Wavelet Transform}

Wavelet transform allows an image to be described in terms of a coarse overall shape plus details that range from broad to narrow. It is a special case of sub band transform with a filter bank being the basic building block. The elements of the filter bank are decomposition filter, reconstruction filter, downsampler and up-sampler [19-22]. Bi-orthogonal wavelet uses one basis function for decomposition and another for reconstruction. They exhibit linear phase property. It satisfies both the requirements of watermarking such as symmetry and perfect reconstruction. It can enhance imperceptibility and robustness of the watermark against intentional and unintentional attacks [23-24].

\subsection{Human Visual System (HVS)}

The sensitivity of human vision is different in various spatial frequencies (frequency bands). The mathematical models of how humans see the world is enabled by HVS research [25]. Studies have shown that a number of factors affect noise sensitivity of human eye like luminance, frequency band, texture, proximity to an edge. Human eye is less sensitive to noise in high resolution bands, bands having orientation of $\pm 45^{\circ}$, to noise in areas where brightness is high or low and to noise in highly textured areas [26-27]. The proposed scheme uses human visual characteristics to exploit human eye limitation to embed strong watermark which also maintains perceptual invisibility using bi-orthogonal wavelet transform.

\subsection{Fuzzy Inference System (FIS)}

Fuzzy set theory has potential capability to represent input and output relationships of dynamic systems. Conventionally, determination of a wavelet tree to be a zero tree was done by comparing the sub-band coefficients uniformly with a fixed threshold value. All coefficients of a wavelet tree less than the threshold value are set as insignificant. On the hand, all coefficients of wavelet tree slightly greater than the threshold value are set as significant. To provide logical reasoning with the uncertain information, a fuzzy inference system is required to be developed. The HVS parameters are combined using FIS to adaptively embed different strength of watermark in different regions by modifying wavelet coefficients [28-29].

\section{EMBEDDING PROCESS}

The watermark used for embedding is a gray scale logo image, which is small compared to the size of host image. The steps for embedding are shown in fig. 1 .

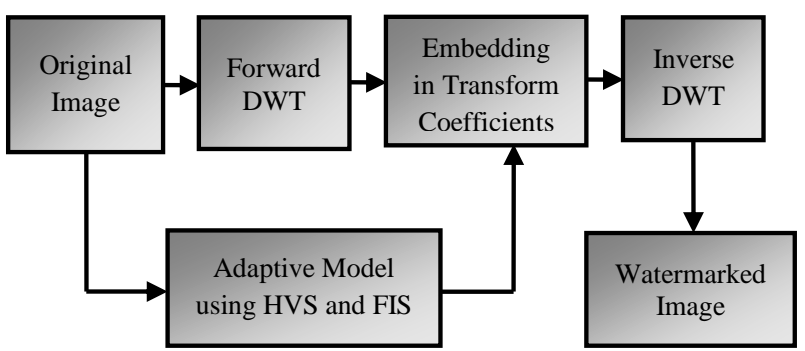

\section{Fig.1 Watermark Embedding Process}

The embedding process is as follows:

1. Decomposition of original image by using bi-orthogonal wavelet transform resulting in four sub-bands $\mathrm{LL}_{1}, \mathrm{LH}_{1}$, $\mathrm{HL}_{1}$, and $\mathrm{HH}_{1}$.
2. Further, decomposition of $\mathrm{LL}_{1}$ sub-band into $\mathrm{LL}_{2}, \mathrm{LH}_{2}$, $\mathrm{HL}_{2}$, and $\mathrm{HH}_{2}$ and $\mathrm{LL}_{2}$ in turn into sub-bands $\mathrm{LL}_{3}, \mathrm{LH} 3$, HL3, and HH3.

3. Obtaining wavelet coefficients for each sub-band and modifying coefficients using HVS and FIS based adaptive model.

4. Selection and calculation of HVS parameters such as brightness, texture and contrast for each sub-band by using approximate sub-band of the respective level.

5. Insertion of watermark in the form of binary logo, sign, text or a combination thereof by modifying the significant wavelet coefficients at each level of decomposition structure.

6. Applying inverse wavelet transform to get watermarked image and calculation of PSNR values at different embedding strengths for imperceptibility analysis of the proposed scheme.

\section{EXTRACTION PROCESS}

The steps for extracting watermark from watermarked image are shown in fig. 2 .

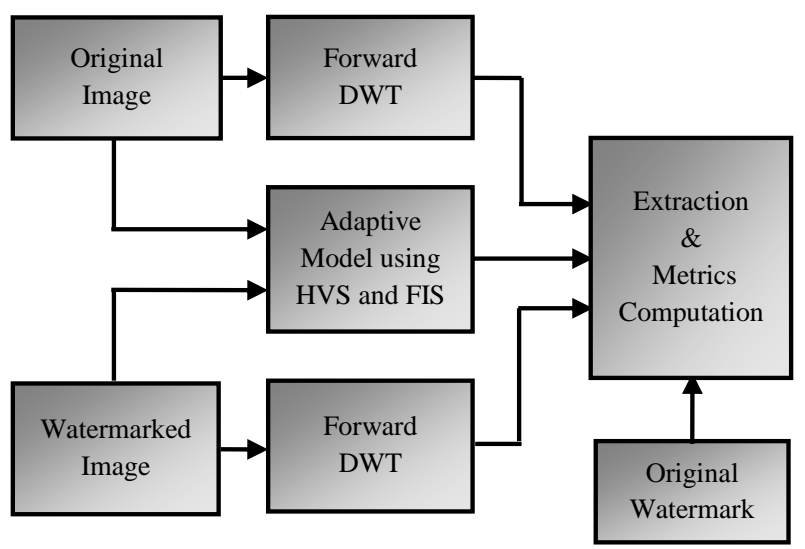

Fig.2 Watermark Extraction Process

1. Realization of wavelet transforms on watermarked image and host image.

2. Extraction of watermark based on adaptive model using HVS and FIS

3. Calculation of correlation coefficient (CRC) for robustness analysis of the proposed scheme and testing watermarked image is for various geometric and nongeometric attacks [30].

\section{RESULTS AND DISCUSSION}

Performance of the proposed scheme is tested on various images as well as various watermarks. The host image is decomposed into ten sub-bands. The watermark image is embedded in the 10 sub-bands based on fuzzy inference system. The FIS accepts three HVS inputs and applies fuzzy rules to determine strength of watermark. The original and watermarked Lena images are shown in Fig. 3a and Fig. 3b respectively. It can be observed that there is no perceptual degradation between original and watermarked image. Fig. 4 and 5 shows original and watermarked kid and fruit images. There is no perceptual degradation in watermarked images. 


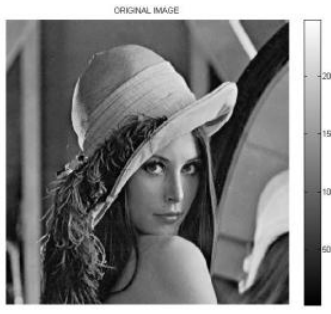

Fig.3a Original Lena

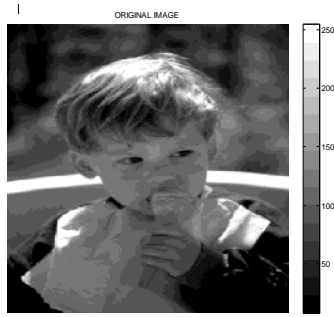

Fig.4a Original Kid

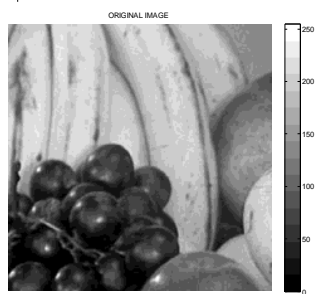

Fig.5a Original Fruit

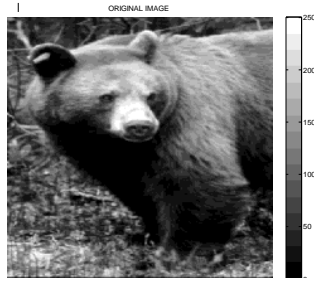

Fig.6a Original Bear

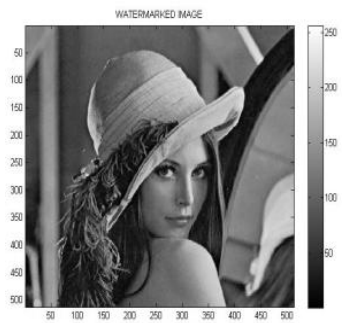

Fig. 3b Watermarked Lena

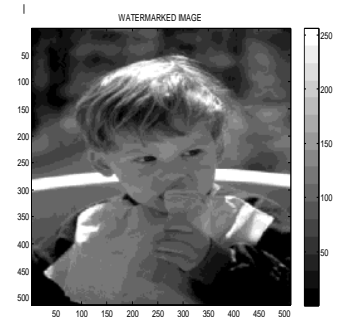

Fig.4b Watermarked Kid

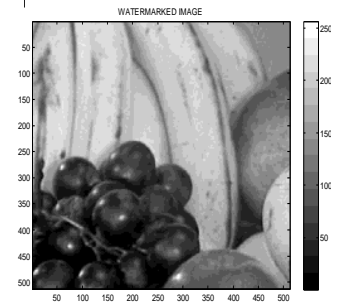

Fig. 5b Watermarked Fruit

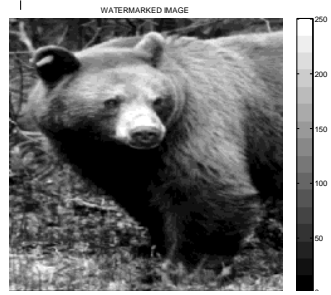

Fig. 6b Watermarked Bear

Fig. $6 \mathrm{a}$ and $6 \mathrm{~b}$ depict original logo image and extraced logo image. Fig. 7 shows example of another watermark embedded and extracted. One can easily identify ewxtracted logo and see no significant visual degradation between embedded watermark and extracted watermark.

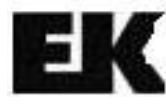

Fig. 7a Embedded

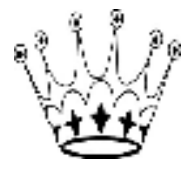

Fig. 8a Embedded Watermark-2

Several signal processing and geometric transformation attacks are performed on these images. Table 1 shows the result for test image 'Lena' with watermark-2. It shows salt and pepper noise attack on original Lena image for various noise density levels and extracted watermark from the attacked Lena image. Retrieved logo images are still recognizable visually even though quality of the attacked image has been severely distorted.

Table1.Watermark Extraction from Attacked Image

\begin{tabular}{|c|c|c|}
\hline Type of Attack & Attacked Image & $\begin{array}{c}\text { Extracted } \\
\text { Watermark }\end{array}$ \\
\hline $\begin{array}{l}\text { Salt \& Pepper Noise } \\
\text { (Noise Density 0.01) }\end{array}$ & PSNR=24.73dB & CRC=0.94 \\
\hline $\begin{array}{l}\text { Salt \& Pepper Noise } \\
\text { (Noise Density 0.03) }\end{array}$ & PSNR=20.64dB & CRC=0.90 \\
\hline & & \\
\hline
\end{tabular}

Fig. 9 relation between PSNR and salt and pepper noise density graphically. The original image is sufficiently degraded with noise density of 0.1 and corresponding PSNR value of $15.34 \mathrm{~dB}$. Graph shows degradation of PSNR value with respect to noise density.

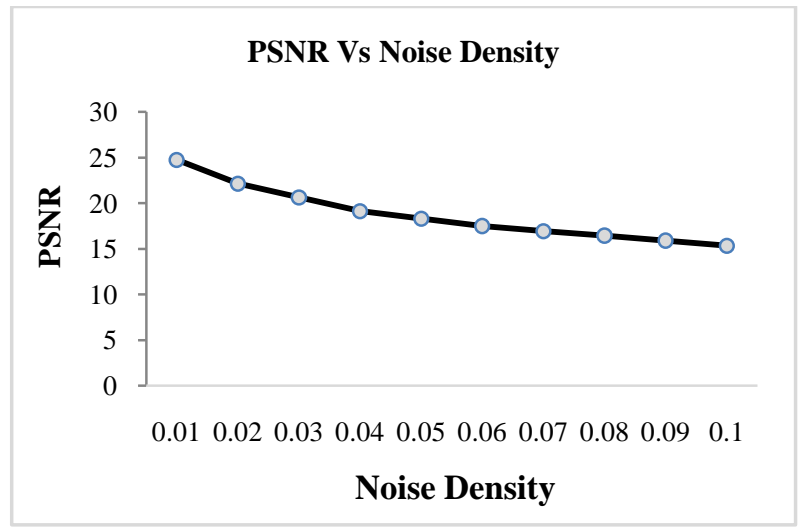

Fig. 9 Relation between PSNR and Salt \&Pepper Noise Density

Fig. 10 shows relation between CRC and salt and pepper noise density graphically. The minimum CRC value is 0.606 for maximum degradation with PSNR value of $15.34 \mathrm{~dB}$ thus allowing extraction and detection of embedded watermark successfully. 


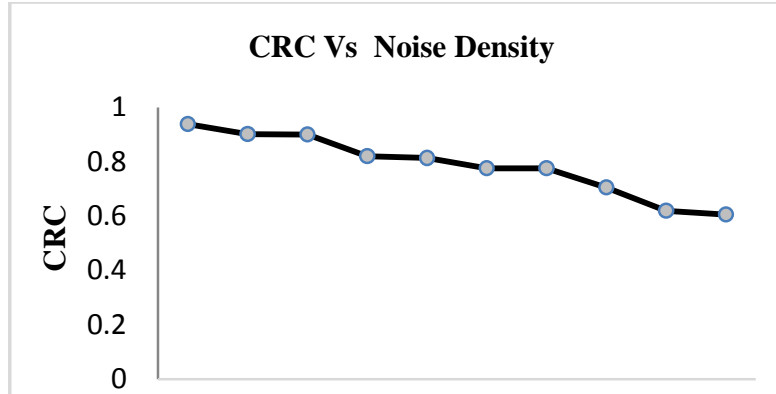

$\begin{array}{llllllllll}0.01 & 0.02 & 0.03 & 0.04 & 0.05 & 0.06 & 0.07 & 0.08 & 0.09 & 0.1\end{array}$

Fig. 10 Relation between CRC and Salt \&Pepper Noise Density

It is observed that proposed scheme can accurately model regions in which watermark insertion is performed, as it takes full advantage of the image adaptive HVS-FIS strategy leading to adaptive image watermarking. The watermark is constrained within the highly non-uniform regions of the image, in order to preserve imperceptibility and robustness criterion.

Table 2 shows results for PSNR for various values of embedding strength values. The results using bi-orthogonal wavelet transform are compared with that of Haar wavelet transform. The table shows comparison of bi-orthogonal wavelet with Haar wavelet.

Table 2. Comparison of PSNR Values for Bi-orthogonal \& Haar Wavelet for Different Embedding Strengths

\begin{tabular}{|c|c|c|}
\hline \multirow{2}{*}{$\begin{array}{c}\text { Embedding } \\
\text { Strength }\end{array}$} & \multicolumn{2}{|c|}{ PSNR (dB) } \\
\cline { 2 - 3 } & $\begin{array}{c}\text { Bi-orthogonal } \\
\text { Wavelet }\end{array}$ & Haar Wavelet \\
\hline 0.5 & 50.24 & 48.44 \\
\hline 1.0 & 44.22 & 42.42 \\
\hline 1.5 & 40.69 & 38.89 \\
\hline 2.0 & 38.20 & 36.39 \\
\hline
\end{tabular}

Fig. 11 shows relation between PSNR values and embedding strength graphically.

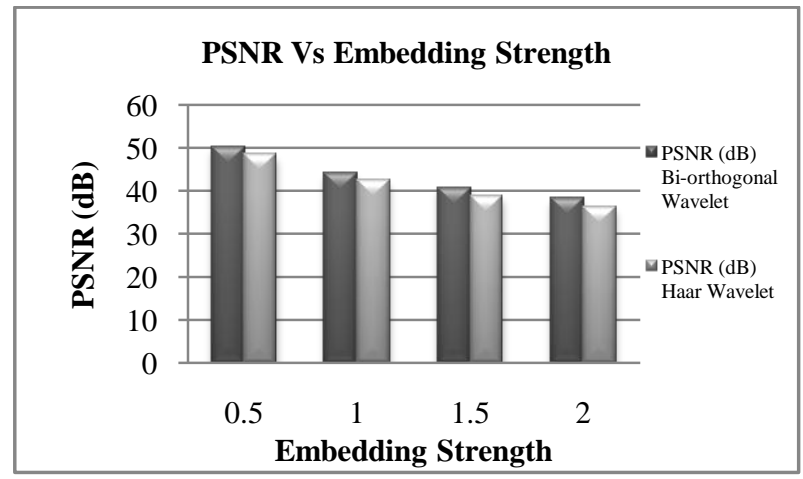

Fig. 11 PSNR \& Embedding Strength for Bi-orthogonal \& Haar Wavelet

The minimum value of PSNR required for imperceptibility is $35 \mathrm{~dB}$. The watermarked Lena image has PSNR values above this minimum level which means that modification of original image almost imperceptible to the human vision. The graph gives comparative study of Bi-orthogonal and Haar wavelet. It shows better PSNR values for bi-orthogonal wavelet. A Harr wavelet transform is conceptually simple and fast but disadvantage is that it is not continuous.

Table 3 shows comparison of PSNR and CRC values for proposed method with that of DCT method.

Table 3. Comparison of PSNR and CRC Values under Various Attacks for Proposed Method and DCT Method

\begin{tabular}{|c|c|c|c|c|}
\hline \multirow{2}{*}{$\begin{array}{c}\text { Type of } \\
\text { Attack }\end{array}$} & \multicolumn{2}{|c|}{ Proposed Method } & \multicolumn{2}{c|}{ DCT Method } \\
\cline { 2 - 5 } & $\begin{array}{c}\text { PSNR } \\
\text { (dB) }\end{array}$ & CRC & $\begin{array}{c}\text { PSNR } \\
\text { (dB) }\end{array}$ & CRC \\
\hline $\begin{array}{c}\text { Gaussian } \\
\text { Noise }\end{array}$ & 30.66 & 0.77 & 22.63 & 0.70 \\
\hline $\begin{array}{c}\text { JPEG } \\
\text { Compression }\end{array}$ & 39.14 & 0.99 & 38.03 & 0.98 \\
\hline Cropping & 18.35 & 0.89 & 12.05 & 0.85 \\
\hline
\end{tabular}

PSNR values for proposed method are better compared with DCT method which shows better invisibility. Better values of CRC indicate better robustness of the proposed scheme improving performance.

\section{CONCLUSION}

A bi-orthogonal wavelet based image watermarking technique is presented. Watermark is embedded into wavelet coefficients which are selected based on the weight factors calculated by exploiting HVS characteristics and fuzzy inference system. Biorthogonal wavelet shows better values of PSNR as these are symmetric wavelet and result in linear phase transfer of filter coefficients. Bi-orthogonal Wavelet based adaptive image watermarking using human visual system and fuzzy inference system provides a better trade off between two contradictory requirements of image watermarking such as imperceptibility and robustness. Symmetry and perfect reconstruction properties of bi-orthogonal wavelet make it suitable for imperceptible and robust image watermarking applications.

\section{REFERENCES}

[1] Santi P. Maity and Seba Maity, "Multistage Spread Spectrum Watermark Detection Technique using Fuzzy Logic”, IEEE Signal Processing Letters, Vol.16, No.4, 2009, pp. 245-248.

[2] Victor V., Guzman, Meana, "Analysis of a Waveletbased Watermarking Algorithm", IEEE Proceedings of the International Conference on Electronics, Communications and Computer,2004, pp. 283-287.

[3] Zhao Dawei, Chen Guanrong, Liu Wenbo, "A Chaosbased Robust Wavelet-domain Watermarking Algorithm", IEEE International Conference on Multimedia and Expo (ICME), 2004.

[4] N. Kaewkamnerd and K.R. Rao, "Wavelet Based Image Adaptive Watermarking Scheme", IEEE Electronic Letters, Vol. 36,Feb. 2000, pp.312-313.

[5] Vaishali S. Jabade and Sachin R. Gengaje "Literature Review of Wavelet Based Digital Image Watermarking Techniques", International Journal of Computer Applications (0975 - 8887)Volume 31- No.1, October 2011, pp. 28-35. 
[6] D. Kundur and D. Hatzinakos, "Digital Wateramrking using Multiresolution Wavelet Decomposition", Proceedings, IEEE International Conference Acoustic, Speech, Signal Processing,Vol. 5,1998, pp. 2969-2972.

[7] Miang Zuang Zang, Na Zang and Jian Guo Jiang, “An Adaptive Digital Watermarking Algorithm Based on Balanced Multi-wavelet", Proceedings, Fifth IEEE International Conference on Information Assurance ans Security,2009, pp. 243-246.

[8] Hey Jiang and Liu Jianjun, “A Digital Watermarking Algorithm for DEM Image Based on Stationary Wavelet Transform", Fifth IEEE International Conference on Information Assurance and Security, 2009, pp. 221-224.

[9] Peng Liu, Zhizhong Ding, “A Blind Image Watermarking Scheme Based on Wavelet tree Quantization", Second IEEE International Symposium on Electronic Commerce and Security, 2009, pp. 218222

[10] Daxing Zhang, Zhigeng Pan and Haihua Li, "A Contourbased Semi-fragile Image Watermarking Algorithm in DWT Domain", Second IEEE International Workshop on Education Technology and Computer Science, 2010, pp. 228-231.

[11] Wei Cao, Yixin Yan, Shengming Li, "Robust Image Watermarking Based on Singular Value Decomposition in DT-CWT Domain", IEEE International Workshop on Imaging Systems and Techniques, IST, 2009.

[12] Lin Zhuang "Multipurpose Digital Watermarking Algorithm Based on Morphological Wavelet Transform", IEEE International Conference on Communications and Mobile Computing, 2009, pp. 396-400.

[13] Peter Meerwald, Christian Koidl, and Andreas Uhl, "Attack on Watermarking Method Based on Significant Difference of Wavelet Coefficient Quantization", IEEE Transactions on Multimedia, Vol. 11, No. 5, August 2009, pp. 1037-1041.

[14] Der-Chyuan Lou , Hao-Kuan Tso, Jiang-Lung Liu "A Copyright Protection Scheme For Digital Images Using Visual Cryptography Technique", Computer Standards and Interfaces 29, 2007, pp.125-131.

[15] Santa Agreste, Guido Andaloro, Daniela Prestipino, Luigia Puccio, "An Image Adaptive Wavelet Based Watermarking of Digital Images", Journal of Computational and Applied Mathematics, 2006, pp. 1-9.

[16] Wen-Nung Lie, Guo-Shiang Lin, Sheng-Lung Cheng, "Dual Protection of JPEG Image Based on Informed Embedding And Two Stage Watermark Extraction Techniques", IEEE Transactions on information forensics and security, Vol. I, 2006, pp. 330-341.

[17] Enpingli, Huaqing Liang, Xinxin Niu, "Image Watermarking Scheme Based On Wavelet Tree Quantization Robust To Geometric Attacks", IEEE Proceedings of Sixth World Congress on Intelligent Control and Automation, pp. 10256-10260.

[18] Said E. El-Khamy, Fellow IEEE, Mona I. Lotfy and Rowayda A. Sadek, "A Block Based Wavelet Watermarking Technique for Copyright Protection and Authentication", IEEE Transactions, Vol.1, 2003, pp. 9093.
[19] Ming-shing Hsieh, "Image Watermarking based on Fuzzy Inference Filter", IEEE Proceedings of the International Conference on Machine Learning and Cybernetics, Baoding, 2009, pp.3058-3063.

[20] Zhiquan Lu and Xiao-Ping Zhang,"Robust Image Watermarking Based on the Wavelet Contour Detection", IEEE Transactions, Vol.2, 2005, pp. 11651168.

[21] Yusnita Yusof, Othman Khalifa, "Imperceptibility and Robustness Analysis of DWT- Based Digital Image Wateramrking", Proceedings of the International Conference on Computer and Communication Engineering, 2008, pp. 1325-1330.

[22] M.F. Fahmy and G. Fahmy, "A Quasi Blind Watermark Extraction of watermarked Natural Preserve Transform Images", IEEE Ineternational Conference on Image Processing,2009, pp.3665-3668.

[23] Dr. M.A. Dorairangaswamy, B. Padmavathi, "An Effective Blind Watermarking Scheme for Protecting Rightful Ownership of Digital Images", IEEE, TENCON, 2009, pp. 1-6.

[24] Ming-Xiang Zang, Na Zang, Jian-guo Jiang, “An Adaptive Digital Watermarking Algorithm Based on Balanced Multi-wavelet", Fifth international Conference on Information Assuirance and Security, 2009, pp. 243246.

[25] Gui Xie (2004),"Robust Wavelet-Based Blind Image Watermarking Against Geometrical Attacks", IEEE International Conference on Multimedia and Expo (ICME),Vol.3, 2004, pp. 2051-2054.

[26] B.N. Chatterji, Manesh Kokare, A. Adhipathi Reddy, Rajib Kumar Jha., "Wavelets for Content Based Image Retrieval and Digital Watermarking for Multimedia Applications", IEEE Transactions, Vol.2, 2003, pp. 812816.

[27] A. Adhipati Reddy, B. N. Chatterji, "A New Wavelet Based Logo Watermarking Scheme", Science Direct, Pattern Recognition Letters, 26, 2004, pp.1019-1027.

[28] Ming-shing Hsieh, "Image Watermarking based on Fuzzy Inference Filter", IEEE Proceedings of the International Conference on Machine Learning and Cybernetics, Baoding, 2009, pp. 3058-3063.

[29] Chip Hong Chang and Mingyan Zhang, "Fuzzy-Art based adaptive digital watermarking scheme", IEEE Transactions on Circuits and Systems for Video Technology, Vol. 15, no. 1, 2005, pp. 65-81.

[30] Vaishali S. Jabade and Sachin R. Gengaje, "Logo based Image Copyright Protection using Discrete Wavelet transform and Fuzzy Inference System", International Journal of Computer Applications, Vol. 58- No. 10, November 2012, pp. 22 to 28 